\title{
ON TWO PROBLEMS OF H. NEUMANN AND J. WIEGOLD ABOUT LINKED PRODUCTS OF GROUPS
}

\author{
S.V. IVANOV and A.Yu. OL'ShanskiI
}

The authors give affirmative answers to two questions of $H$. Neumann and Wiegold on linked embeddings of groups.

1.

In the paper [3] of Hanna Neumann and J. Wiegold, a concept of linked product of groups was introduced and studied. A group $L$ is called the linked product of groups $G_{1}$ and $G_{2}$ with kernels $K_{1} \unlhd G_{1}$ and $K_{2} \unlhd G_{2}$ (being normal subgroups of $G_{1}$ and $G_{2}$ respectively) if the following conditions are satisfied:

(i) $L$ contains $G_{1}$ and $G_{2}$ as subgroups and $G_{1} \cap G_{2}=1$ in $L$.

(ii) $L=\left\langle G_{1}, G_{2}\right\rangle$, that is, $L$ is generated by subgroups $G_{1}$ and $G_{2}$.

(iii) The normal closure $G_{1}^{L}$ of $G_{1}$ in $L$ meets $G_{2}$ in $K_{2}$ and the normal closure $G_{2}^{L}$ of $G_{2}$ meets $G_{1}$ in $K_{1}$.

The question about the existence of a linked product of given groups $G_{1}$ and $G_{2}$ with given kernels $K_{1}$ and $K_{2}$ was discussed in detail in $[3,4]$ and answered in the affirmative in a number of special cases. These cases gave the authors of [3] a background for the following conjecture: A linked product $L$ exists whatever nontrivial groups $G_{1}, G_{2}$ and their kernels $K_{1}, K_{2}$ are chosen except for the case where $G_{1}$ and $G_{2}$ have order 2 (we write $\left|G_{1}\right|=\left|G_{2}\right|=2$ ) and one of the kernels $K_{1}$ and $K_{2}$ has order 2 while the order of the other is 1 . It is easy to see that in the latter case there exists no linked product.

The following theorem confirms the conjecture.

THEOREM 1. Let $G_{1}$ and $G_{2}$ be nontrivial groups, $K_{1} \unlhd G_{1}$ and $K_{2} \unlhd G_{2}$ their normal subgroups and neither of the two following possibilities holds:

(i) $\left|G_{1}\right|=\left|G_{2}\right|=\left|K_{1}\right|=2$ and $\left|K_{2}\right|=1$

(ii) $\left|G_{1}\right|=\left|G_{2}\right|=\left|K_{2}\right|=2$ and $\left|K_{1}\right|=1$.

Received 23 September 1991

Copyright Clearance Centre, Inc. Serial-fee code: 0004-9729/92 \$A2.00+0.00. 
Then there exists a linked product $L$ of the groups $G_{1}$ and $G_{2}$ with kernels $K_{1}$ and $K_{2}$.

In [3], Neumann and Wiegold posed another problem: can one find, given nontrivial groups $G_{1}$ and $G_{2}$, a simple group $S=\left\langle G_{1}, G_{2}\right\rangle$ in which $G_{1} \cap G_{2}=1$. (This is a stronger form of the previous question, since $S$ is a simple linked product of $G_{1}$ and $G_{2}$ with kernels $G_{1}$ and $G_{2}$.) Clearly, the question is answered in the negative if $\left|G_{1}\right|=\left|G_{2}\right|=2$. It appears that this is the single such case.

THEOREM 2. Let $G_{1}$ and $G_{2}$ be nontrivial groups the product of whose orders is not 4 .

(a) Then there exists a simple linked product $L$ of the groups $G_{1}$ and $G_{2}$ (whose kernels are of course $G_{1}$ and $G_{2}$ ).

(b) Additionally suppose that $G_{1}$ and $G_{2}$ are finitely generated. Then there are uncountably many non-isomorphic simple linked products of groups $G_{1}$ and $G_{2}$.

Both theorems can be proved simultaneously, using a technique of graded diagrams as treated in [5]. However the first theorem has a very short proof using arguments of ordinary small cancellation theory. We present this proof which, moreover, helps in understanding the idea of the proof of the second theorem. To prove the last theorem we need [5] and additional arguments of [1] or [6] in the case where the groups $G_{1}$ and $G_{2}$ have involutions. We choose [1] as it gives a shorter way. So our exposition in the third section depends heavily on [5] and [1].

2.

In this section, imposing defining relations on the free product $F=G_{1} * G_{2}$, we construct a linked product $L$ of given nontrivial groups $G_{1}$ and $G_{2}$ with kernels $K_{1}$ and $K_{2}$.

One can assume that $\left|G_{1}\right| \geqslant 2$ and $\left|G_{2}\right| \geqslant 3$ and fix elements $a \in G_{1} \backslash 1$ and $b, c \in G_{2} \backslash 1$, where $b c \neq 1$. Also, let $k, l$ be some coprime integers such that $k, l \geqslant 30$. For every $x \in K_{1} \backslash 1$ and $y \in K_{2} \backslash 1$ impose the following relations on $F$ :

$$
\begin{gathered}
\left(b c a b a b^{-1} a^{-1} c^{-1} a^{-1} b^{-1} x\right)^{k}=1 \\
\left(b c a b a b a b^{-1} a^{-1} b^{-1} a^{-1} c^{-1} a^{-1} b^{-1} x\right)^{l}=1
\end{gathered}
$$

and

$$
\begin{gathered}
\left(a b c a c^{-1} a^{-1} b^{-1} a^{-1} y\right)^{k}=1 \\
\left(a b c a c^{-1} a b^{-1} a^{-1} b a^{-1} b^{-1} a^{-1} y\right)^{\prime}=1
\end{gathered}
$$


Let $r_{1}, r_{2}$ denote two arbitrary cyclic permutations of relators (1), (2), (3), (4) or inverse words. We can write $r_{1}=u_{1}^{m}, r_{2}=u_{2}^{n}$ in $F$ where $m, n \geqslant 30$. It is clear that $r_{1}=r_{2}$, if the length $|v|$ (that is, the length of the normal form in the free product $F$ ) of the maximal common prefix $v$ of words $r_{1}$ and $r_{2}$ is greater than $2 \max \left(\left|u_{1}\right|,\left|u_{2}\right|\right)$. Otherwise $|v| \leqslant(1 / 9) \min \left(\left|r_{1}\right|,\left|r_{2}\right|\right)$, as $k, l \geqslant 30$. Therefore the condition $C^{\prime}(1 / 6)$ holds for relators (1), (2), (3), (4). Hence the normal closure $N$ of all relators (1), (2), (3), (4) in $F$ satisfies the following conditions: $G_{1} \cap N=1, G_{2} \cap N=1$ and $G_{1} N \cap G_{2} N=1$ (see [2, Chapter 5]), that is $G_{1} \cap G_{2}=1$ in $L=F / N$.

By the definition of the relators (1), (2), (3), (4), the subgroup $N$ is contained in the kernel of the natural homomorphism onto the direct product: $F \rightarrow\left(G_{1} / K_{1}\right) \times\left(G_{2} / K_{2}\right)$. Therefore $G_{2}^{L} \cap G_{1} \leqslant K_{1}$ in $L$. On the other hand, $x^{k} \in G_{2}^{L}$ for each $x \in K_{1}$ in view of (1), as

$$
b c a b a b^{-1} a^{-1} c^{-1} a^{-1} b^{-1} \in G_{2}^{F} .
$$

By the same reasoning (see (2)) $x^{\prime} \in G_{2}^{L}, x \in G_{2}^{L}$, as $(k, l)=1$. Consequently, $G_{2}^{L} \cap G_{1}=K_{1}$. In a similar way, $G_{1}^{L} \cap G_{2}=K_{2}$, and Theorem 1 is proved. Moreover, the construction of the group $L$ is effective by standard small cancellation arguments.

3.

To prove Theorem 2 , we need an infinite increasing sequence of rather large primes $P=\left(p_{1}, p_{2}, \ldots\right)$ (that is, $p_{i}>10^{10}$ for $\left.i=1,2, \ldots\right)$.

Let $L(0)=F=G_{1} * G_{2}$ and the set $R(0)$ of relators of rank 0 be empty.

Defining the sets $R(i)$ and the groups $L(i)$ by induction on $i$, we suppose that $R(i-1)$ and $L(i-1)$ have already been constructed.

Let $M(i)$ be a set of words of length $i$ over the alphabet $\left(G_{1} \cup G_{2}\right) \backslash 1$ maximal with respect to the following properties:

M1. If $u$ and $v$ are different elements of $M(i)$, then $u$ is not conjugate to $v^{ \pm 1}$ in $L(i-1)$.

M2. Any $u \in M(i)$ is not conjugate in $L(i-1)$ to a power of a word $w$ with $|w| \leqslant \max (1, i-1)$.

M3. Each $u \in M(i)$ is not equal in $L(i-1)$ to a product of two elements of order 2.

Then we define $R(i)$ by $R(i)=R(i-1)$ if $M(i)=\emptyset$. Otherwise, $R(i)=R(i-1) \cup$ $\left\{u^{p} \mid u \in M(i)\right\}$, where $p$ is the first prime of the sequence $P$, which was not used at previous steps.

Now we give the group $L(i)$ by defining relators from $R(i)$ :

$$
L(i)=\langle F \| R(i)\rangle .
$$


Since for any $i \geqslant 1$ we have already defined the sets $R(i)$, we can give the following limit group $L(\infty)$ :

$$
L(\infty)=\left\langle F \| R(\infty)=\bigcup_{i=1}^{\infty} R(i)\right\rangle
$$

As in [5, Section 36] it is proved that $G_{1}$ and $G_{2}$ embed properly in $L(\infty)$ and intersect trivially. (The only difference from [5] is the infinity of the set $P$ of exponents of periods $u \in M=\bigcup_{i=1}^{\infty} M(i)$. This does not affect the proof.)

The paper [1] contains a construction of a sequence of elements $E_{1}, E_{2}, \ldots \in F$ (in the case where $\left|G_{1}\right| \geqslant 2,\left|G_{2}\right| \geqslant 3$ ) such that $\left|E_{j}\right| \rightarrow \infty$ if $j$ tends to $\infty$ and the following statement holds.

Lemma 1. Let $q=t_{1} E t_{2} E^{-1}$, where $E=E_{j}$ for some $j=1,2, \ldots$ Assume that the word $q$ is conjugate in $L(\infty)$ to a word $u$ and $\left|t_{1}\right|+\left|t_{2}\right|<0.01|E|$. Then the inequality $|u|<0.01|E|$ involves equalities $t_{1}=t_{2}=u=1$ in the group $L(\infty)$.

As a matter of fact, $[1$, Lemma 6] deals with another group (not with $L(\infty)$ ), and it demands a stronger inequality $|u| \leqslant 1$, but the proof of $[1$, Lemma 6$]$ can be repeated under the hypotheses of Lemma 1 without any change.

Now consider a nontrivial normal subgroup $N$ of $L=L(\infty)$. The assumption that $s^{2}=1$ for every element $s \in N$ gives us one of two inclusions: $N \leqslant G_{1}$ or $N \leqslant G_{2}$ by [5, Lemmas 36.4 and 18.1]. However both inclusions are impossible as $x G_{j} x^{-1} \cap G_{j}=1$ if $j=1,2$ and $x \notin G_{j}$. (See [1, Lemma 4] or [6, Theorem 2, item 4]).

So, one can choose $s \in N$ such that $s^{2} \neq 1$. Let $v$ be any nontrivial element of $F$. Choose $E=E_{j}$ such that $|E|>10^{3} \max (|v|,|s|)$ and consider the word $w=E s E^{-1} v$. Then $w$ represents a nontrivial element of $L$, which is conjugate to a power of some word $u \in M(i)$ for some $i$, as it was explained in [1]. So $w^{p}=1$ in $L$ for some $p \in P$, and $v^{p} \in N$, because $E s E^{-1} \in N$.

One can repeat the previous construction of the word $w$, choosing $E^{\prime}=E_{k}$ such that $\left|E^{\prime}\right|>100 p|u|$. The word $w^{\prime}$ obtained is conjugate to a power $\left(u^{\prime}\right)^{m}$ of some $u^{\prime} \in M\left(i^{\prime}\right)$. Note that $i^{\prime}>i$, because otherwise $\left|E^{\prime}\right|>100 m\left|u^{\prime}\right|$ and $w^{\prime}=1$ in $L$ by Lemma 1.

Therefore $v^{p} \in N$ for $p^{\prime}>p$ also. This shows the inclusion $v \in N$ for every $v \in L$. Consequently, $N=L$, and the simplicity of the group $L$ is proved.

In order to prove part (b) of Theorem 2, consider quotients $L=F / R$ and $L^{\prime}=$ $F / R^{\prime}$ constructed with the help of different sequences $P$ and $P^{\prime}$ of primes. Let $p$ and $p^{\prime}$ be the first primes in $P$ and $P^{\prime}$ to differ. Then for some $i$ and $u \in M(i)$ we have $u^{p}=1$ in $L$ and $u^{p^{\prime}}=1$ in $L^{\prime}$ by definitions of $L$ and $L^{\prime}$. Also $u \neq 1$ in $L$. This can be explained exactly as in [5, Theorem 36.4]. Therefore $R \neq R^{\prime}$, 
and we have an uncountable set of normal subgroups $R \triangleleft F$ such that $L=F / R$ satisfies the conclusion of Theorem 2 . Finally, note that there is only a countable set of homomorphisms of $F$ onto any fixed countable group $L$, because $F$ is a finitely generated group. Consequently, there is an uncountable set of nonisomorphic quotients $L=F / R$.

\section{REFERENCES}

[1] S.V. Ivanov, 'On periodic products of groups' (to appear).

[2] R. Lyndon and P. Schupp, Combinatorial group theory (Springer-Verlag, Berlin, Heidelberg, New York, 1977).

[3] H. Neumann and J. Wiegold, 'Linked products and linked embeddings of groups', Math. Z. 73 (1960), 1-19.

[4] B.H. Neuman and H. Neumann, 'On linked products of groups', Acta Sci. Math. 21 (1960), 197-205.

[5] A.Yu. Ol'shanskii, Geometry of defining relations in groups, (in Russian) (Nauka, Moscow, 1989).

[6] A. Yu. Ol'shanskii, 'On thrifty embeddings of groups', (in Russian), Vestnik Moskovsk. Univ. 2 (1989), 28-34.

Department of Mathematics and Mechanics

Moscow University

Moscow 119899

Russia 